\title{
Transverse myelopathy and radiculomyelopathy associated with pulmonary atypical Mycobacterium infections
}

\author{
V L C White, R Al-Shahi, E Gamble, P Brown, A G Davison
}

\begin{abstract}
Myelopathy is a well recognised but rare association with Mycobacterium tuberculosis infection, but has not been described with atypical mycobacteria. We report two cases of disabling myelopathy in association with pulmonary infection by Mycobacterium kansasii and Mycobacterium malmoense; the myelopathy is presumed to be a para-infectious phenomenon. (Thorax 2001;56:158-160)
\end{abstract}

Keywords: Mycobacterium malmoense; Mycobacterium kansasii; myelopathy

Approximately 50 species within the genus Mycobacterium exist naturally in the environment: they are variously called atypical mycobacteria, environmental mycobacteria, and non-tuberculous mycobacteria. Opportunistic pulmonary infection with atypical mycobacteria may occur in immunocompetent individuals, especially with previously damaged lungs, and disseminated infection is seen in immunocompromised hosts, especially with AIDS. ${ }^{1-3}$

Both Mycobacterium malmoense and Mycobacterium kansasii are environmental mycobacteria. The number of cases of opportunistic mycobacteria has been rising in England and Wales from 1982 to $1994 .^{2}$ The incidence of $M$
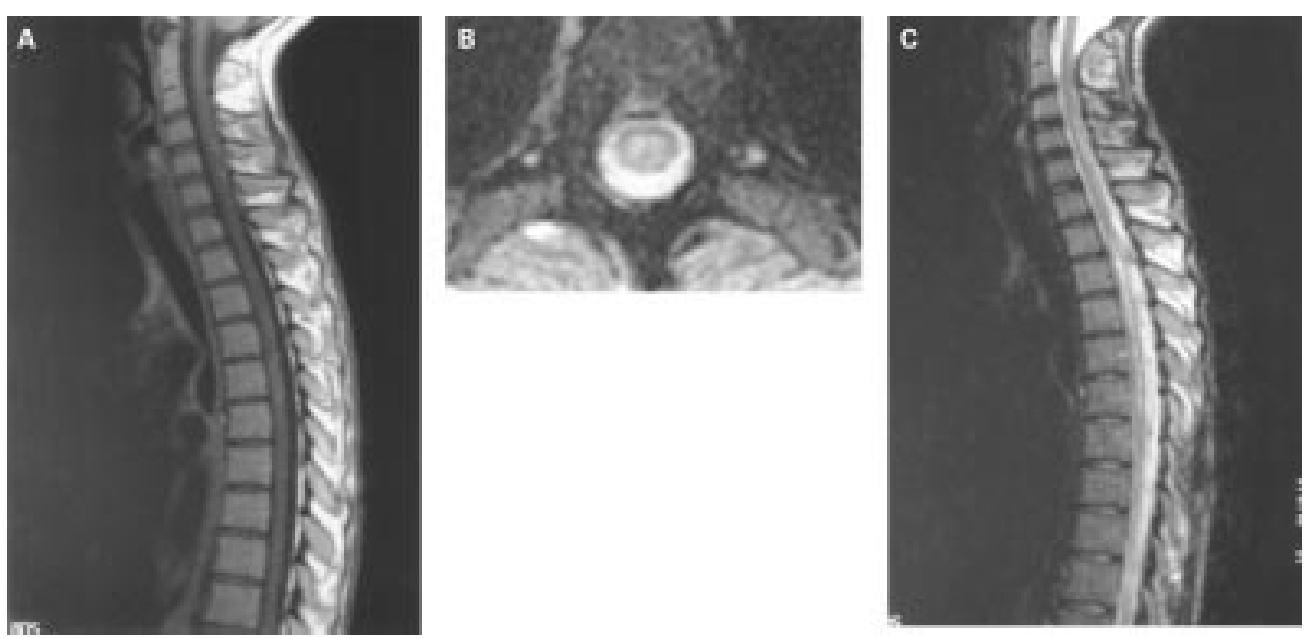

Figure 1 (A) Sagittal T1 weighted magnetic resonance image (MRI) of the cervical and thoracic cord of case 1 demonstrating focal enhancement at the level of T4. (B) Axial MR image of case 1 at the level of T4 showing focal cord enhancement. (C) Sagittal T2 weighted MRI of the cervical and thoracic cord of case 1 revealing high signal within the cord from $T 2$ to $T 5$.

kansasii has, however, remained stable at approximately 50 per year. The number of cases of infection with $M$ malmoense has risen steadily from 1982 and is now approximately 50 per year. $M$ malmoense does not appear to be related to HIV. ${ }^{2}$

Myelopathy has not previously been reported as a complication of environmental mycobacterial infection and other neurological complications are extremely rare. ${ }^{4}$

\section{Case reports}

CASE 1

A 23 year old, right handed computer operator with type 1 diabetes mellitus presented with a four month history of weight loss, productive cough, increasing breathlessness, and a one day history of chest pain. On examination the only abnormalities were coarse inspiratory crackles in both upper zones of the chest and some tenderness to the right of the $\mathrm{T} 8$ spinous process. Routine biochemical and haematological investigations were normal. Chest radiography showed multiple, bilateral, thin walled cavities in the upper zones. Ziehl-Neilson staining of the sputum confirmed the presence of acid and alcohol fast bacilli. HIV antibody test was negative. Treatment was started with rifampicin, isoniazid, and pyrazinamide.

University Hospital Westcliff-on-Sea, Essex, SS0 0RY, UK V L C White

E Gamble

A G Davison

National Hospital for Neurology \&

Neurosurgery, Queen

Square, London WC1N 3BG, UK

R Al-Shahi

P Brown

Correspondence to:

Dr A G Davison

dr.davison@hospital.

southend.nhs.uk

Received 8 March 199 Returned to auth
27 April 1999 Revised version received 28 March 2000 Accepted for publication 10 April 2000 
One month later he presented with a four day history of leg weakness and was unable to walk. He complained of electric shock-like pains in the legs, a two day history of loss of sensation below the umbilicus, and difficulty in passing urine. Clinical examination of the upper limbs was normal. There was lower limb hypertonia with Medical Research Council (MRC) grade 2-3/5 weakness in a pyramidal distribution and extensor plantar responses. There was a sensory level at T6. The cranial nerves and higher function were normal. $\mathrm{He}$ was in urinary retention.

Further investigations showed that autoantibody screen and syphilis serology were negative. Serum B12 levels were normal and the C reactive protein (CRP) level was $80 \mathrm{mg} / \mathrm{l}$. Examination of the cerebrospinal fluid (CSF) revealed a protein level of $0.76 \mathrm{~g} / 1$, glucose $8.4 \mathrm{mmol} / 1$ (serum glucose $8.3 \mathrm{mmol} / \mathrm{l}$ ), white cell count $5 / \mathrm{mm}^{3}$, and there were no oligoclonal bands in the CSF or serum. Gram stain and bacterial culture of the CSF were negative and there was no growth on Lowenstein-Jensen medium at eight weeks. Magnetic resonance imaging (MRI) of the spine showed high signal involving the grey matter and posterior columns at the level of T4 and no abnormalities in the brain (fig 1).

A diagnosis of transverse myelitis was made and the patient was treated with intravenous methylprednisolone $(500 \mathrm{mg})$ daily for three days followed by an oral dose of $100 \mathrm{mg}$ daily which was gradually reduced over 18 weeks. The original sputum cultures grew $M$ kansasii. The antituberculous regime was changed to ethambutol and rifampicin.

Over the following months his respiratory symptoms and chest radiograph gradually improved. There was some recovery of power in the lower limbs such that after six months he was able to weight bear and transfer but remained wheelchair dependent with an indwelling urinary catheter.

CASE 2

A 39 year old, left handed care assistant presented with a 10 day history of pain radiating into the epigastrium associated with nausea and vomiting. She smoked 15 cigarettes per day and had had a non-productive cough for two months without fever. Physical examination was unremarkable. Chest radiography showed patchy consolidation at the right apex and bronchoscopy was arranged as an outpatient.

Within a week she was readmitted with band-like thoracic pain associated with urinary incontinence, paraesthesiae in the legs, and worsening bilateral leg weakness such that she was unable to walk. Examination of the arms was normal but there was hypertonia of the legs with MRC grade $4 / 5$ weakness in a pyramidal distribution, hyperreflexia, and extensor plantar responses. There was a sensory level at T4. The cranial nerves and higher function were normal.

Routine biochemical and haematological investigations were normal, as were immunoglobulins, autoantibodies, CRP, B12, syphi-

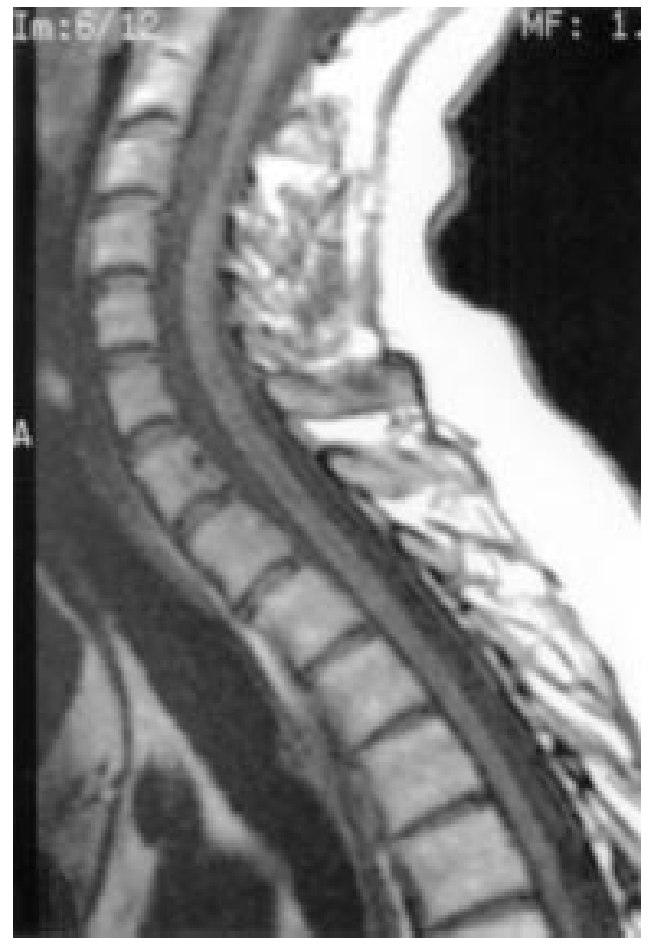

Figure 2 Sagittal T1 weighted magnetic resonance image (MRI) of case 210 weeks after presentation showing atrophy of the cervical cord and probable myelomalacia of the lower cervical and upper thoracic segments.

lis serology, and CD4/CD8 ratio. Examination of the CSF revealed a protein level of $1.4 \mathrm{~g} / \mathrm{l}$, white cell count of $26 / \mathrm{mm}^{3}$, glucose level of $2.9 \mathrm{mmol} / 1$ (serum glucose $5.9 \mathrm{mmol} / \mathrm{l}$ ) with negative Gram and Ziehl-Neilson stains and there was no growth on Lowenstein-Jensen medium at six weeks. The CSF initially showed intrathecal synthesis of a solitary IgG clone but during the illness she developed evidence of intrathecal synthesis of several IgG clones. Bone scan was normal. MRI showed increased signal intensity in the lower cervical and upper thoracic spinal cord and no abnormalities in the brain on repeated examination over two years.

A diagnosis of transverse myelitis was made and the patient was treated with daily intravenous methylprednisolone ( $1 \mathrm{~g})$ for three days with some improvement in leg power. Bronchoscopic lavage showed acid-fast bacilli and the patient was started on antituberculous medication. Subsequent culture of the lavage fluid grew $M$ malmoense and she was treated with isoniazid, rifampicin, pyrazinamide, ethambutol, and streptomycin.

There were three subsequent neurological relapses over the next 14 months, the second of which caused bilateral arm weakness and an episode of acute respiratory failure requiring six weeks' mechanical ventilation. At this stage signal changes within the cord on MRI extended to the craniocervical junction and the medulla oblongata with swelling and irregular contrast enhancement. Each relapse was treated with intravenous methylprednisolone with mild improvement but residual disability.

Eighteen months after presentation she had a spastic quadriparesis with MRC grade 2/5 
weakness in a pyramidal pattern in the legs and grade $4 / 5$ weakness in the arms with superimposed, right-sided wasting and weakness of trapezius, serratus anterior, infraspinatus, supinator and small hand muscles. There was evidence of action induced myoclonus of the right fingers. She had a sensory level at C3 and a forced vital capacity of 2.4 litres. Visual acuities in both eyes were $6 / 6$ with normal colour vision and no evidence of afferent pupillary defects. Cranial nerves and higher cortical function were normal. The cervical spinal cord appeared atrophic on MRI, maximal at C3 (fig 2 ). She had abnormal somatosensory evoked potentials and nerve conduction studies from both upper limbs suggesting a lesion in the large fibre sensory pathways at the level of the preganglionic C6/7 dorsal roots. Visual evoked potentials of both eyes were attenuated, but not delayed, compatible with bilateral axonal optic neuropathy. She has remained clinically stable for 30 months.

\section{Discussion}

Transverse myelopathy has never been reported in association with $M$ malmoense nor $M$ kansasii although $M$ tuberculosis is known to cause a myelopathy by direct infection, arachnoiditis, compression, and as a para-infectious process in association with pulmonary tuberculosis. $^{5-7}$ Both $M$ malmoense and $M$ kansasii are environmental pathogens, the former being found in the soil and the latter in water and is able to colonise piped water supplies. They are also found as contaminants in culture. They are recognised as pulmonary pathogens, particularly in patients with previously damaged lungs. ${ }^{1}$ Healthy people may also be affected, more commonly in middle aged or elderly individuals. They also cause disease in lymph nodes, skin, gut and bursae. ${ }^{8}$ Disseminated infection is now seen in immunocompromised hosts. Human to human spread is not thought to occur. ${ }^{9}$ The recommended treatment is with multiple antituberculous drugs which for $M$ malmoense includes ethambutol and lasts 24 months; for $M$ kansasii regimes should include rifampicin and ethambutol for nine months and isoniazid for three or nine months. ${ }^{310}$

The first patient developed a transverse myelitis some weeks after the initial pulmonary infection was diagnosed and treatment started. The second patient had a combination of a relapsing myelopathy, radiculopathy, and a subclinical optic neuropathy. The myelopathy developed before antituberculous chemotherapy was commenced, although the optic neuropathy may have developed subsequently. Ethambutol is well known to cause retrobulbar neuritis and isoniazid rarely causes optic neuropathy; they may both cause a peripheral neuropathy but myelopathy has not been reported. It is assumed that these myelopathies were para-infectious in origin in the absence of evidence of active tuberculous infection of the spinal cord or a direct relation to treatment.

In three cases of acute myelopathy associated with pulmonary tuberculosis that have undergone post-mortem examination ${ }^{5}$ the myelopathy had been progressive or relapsing (although only one patient received corticosteroids) and demyelination of the white and grey matter was found in the spinal cord of all three patients. Another similar case ${ }^{6}$ showed clinical improvement after steroid therapy. In a small series of patients with neuromyelitis optica (myelopathy and optic neuropathy) and pulmonary tuberculosis ${ }^{7}$ there was no visual improvement; five of the eight patients showed some degree of amelioration of the spinal cord symptoms but the prognosis was worse than in idiopathic transverse myelitis. The CSF findings in all the reported cases were similar to those of our two patients - increased protein with a pleocytosis and occasionally a low glucose level-the only consistent finding being failure to isolate Mycobacterium spp from the CSF. The underlying mechanism for such acute necrotic myelopathies is unclear but it has been postulated that tuberculous infection might amplify the body's immune response to myelin damage through an adjuvant effect or through shared epitopes on myelin basic protein and tubercle bacillus. ${ }^{5}$ The initial development of oligoclonal IgG in the CSF of case 2 supports the hypothesis that tuberculous infection triggers an intrathecal immune response.

1 Zaugg M, Salfinger M, Opravil M, et al. Extrapulmonary and disseminated infections due to Mycobacterium malmoense: case report and review. Clin Infect Dis 1993;16:5409 .

2 Lamden K, Watson JM, Knerer G, et al. Opportunistic mycobacteria. Communicable Dis Rep 1996;6:147-56.

3 Grange JM, Girling DJ, Davies PDO. Disease caused by environmental mycobacteria. In: Weatherall DJ, Ledingham JMG, Warrell DA, eds. Oxford textbook of medicine. Oxford: Oxford University Press, 1996:664-7.

4 Prince $\mathrm{H}$, Ispahani P, Baker M. A Mycobacterium malmoense infection of the hand presenting as carpal tunnel syndrome. f Hand Surg $\operatorname{Br} 1988 ; 13: 328-30$.

5 Hughes RA, Mair WG. Acute necrotic myelopathy with pulmonary tuberculosis. Brain 1977;100:223-38.

6 Reid AC, Bone I. Myelopathy during treatment for pulmonary tuberculosis. Postgrad Med f 1980;56:511-2.

7 Silber MH, Willcox PA, Bowen RM, et al. Neuromyelitis optica (Devic's syndrome) and pulmonary tuberculosis. Neurology 1990;40:934-8.

8 American Thoracic Society. Diagnosis and treatment of disease caused by nontuberculous mycobacteria. Am f Respir Crit Care Med 1997;156:S1-25.

9 Hoffner SE. Pulmonary infections caused by less frequently encountered slow-growing environmental mycobacteria. Eur $\mathcal{F}$ Clin Microbiol Infect Dis 1994;13:937-41.

10 Research Committee of the British Thoracic Society. Mycobacterium kansasii pulmonary infection: a prospective study of the results of nine months of treatment with rifampicin and ethambutol. Thorax 1994;49:442-5. 